\title{
Análisis del conocimiento sobre el ciclo del agua en dos actividades en Primaria
}

Ana Aragüés Díaz. IES Valle del Ebro

0000-0002-8228-0813

Recepción: 16.11.2019 | Aceptado: 29.11.2019

Correspondencia a través de ORCID: Ana Aragüés Díaz

0000-0002-8228-0813

Citar: Aragüés Díaz, A. (2019). Análisis del discurso del aula en dos actividades del Ciclo del Agua en Primaria. REIDOCREA, 8, 458-466.

Resumen: Desde una perspectiva basada en que gran parte del aprendizaje surge de las interacciones sociales producidas dentro del aula, el presente trabajo se focaliza en analizar del discurso de un aula de Primaria durante la realización de dos actividades sobre el ciclo del agua. El análisis se centra en examinar el conjunto de ideas expresadas. Los resultados muestran cómo la aportación de datos, a través del uso de un dispositivo, así como la búsqueda de un consenso en las respuestas del alumnado de Primaria permite a los estudiantes confrontar y establecer ideas través del debate.

Palabras clave: Educación básica I Enseñanza de las ciencias

Analysis of knowledge about the water cycle in two activities in Primary

Abstract: From a perspective based on the great part of the learning of the social interactions produced within the classroom, the present work focuses on the analysis of the discourse of a Primary classroom during the realization of two activities on the water cycle. The analysis focuses on examining the set of ideas expressed. The results indicated how the contribution of data, through the use of a device, as well as the search for a consensus in the responses of Primary students allows students to confront and establish ideas through debate.

Keywords: Elementary Education I Science Education

\section{Introducción}

Una enseñanza de las ciencias que permita adquirir las competencias propias de los científicos es una idea ampliamente compartida por la comunidad educativa (Vílchez y Bravo, 2015). Este enfoque pone de manifiesto la necesidad de integrar la teoría y la práctica cuando se pretende que los alumnos adquieran un determinado conocimiento (Nebot y Márquez, 2014). El énfasis de esta visión del aprendizaje radica en aplicar un modelo didáctico que facilite oportunidades al alumnado para desarrollar procesos tales como plantear preguntas, experimentar o razonar. Autores del campo de la didáctica de las ciencias señalan que, aunque no siempre sea una tarea fácil, una forma de aplicar este modelo didáctico es mediante de la inclusión de actividades prácticas (Rivero, Martín del Pozo, Solís, Azcarate y Porlán, 2017).

En concreto, en el modelo del ciclo del agua tan sólo pueden ser reproducidos aspectos parciales del mismo y tampoco todos ellos (ej. la precipitación en forma de nieve). Además, se suma la dificultad de que precisa del manejo de varios submodelos como son un modelo fisicoquímico de los cambios de estado, así como un modelo de ser vivo cuando se habla, por ejemplo, de cómo las plantas absorben agua del suelo. Así pues, la complejidad en el estudio de este contenido ha favorecido a que tradicionalmente se haya abordado de una forma meramente descriptiva. No es de extrañar, por tanto, que de ello resulte un aprendizaje más bien superficial sobre el mismo (Smith, Wiser, Anderson y Krajcik, 2006). Son numerosas las publicaciones que hacen referencia a un elevado número de ideas alternativas con relación al ciclo del agua que además parecen persistir a lo largo de toda la etapa educativa en las mentes de los alumnos (Cardak, 2009; de Miguel, Lado, Martínez, Leal y García, 2009). Desde 
nuestra perspectiva el aprendizaje viene dado, en gran medida, por las distintas interacciones sociales que se dan dentro del aula (Bruner, 1984), de manera que una posible forma de salvar estas ideas en las mentes de los estudiantes puede ser mediante actividades en las que se enfrenta al alumnado a situaciones problemáticas (Marquez, Izquierdo y Espinet, 2003).

\section{El ciclo del agua en Primaria}

El ciclo del agua es un contenido que engloba distintas áreas de conocimiento como son la Química, la Física, la Biología y la Geología en el que las ideas tratadas requieren un alto nivel de complejidad (Márquez, Izquierdo y Espinet, 2006). El elevado número de procesos simultáneos como son los cambios de estado, los fenómenos meteorológicos, o los cambios de densidad determinan el número de ideas alternativas que algunos estudios describen en las mentes del alumnado (Calvo, Reyero, Vidal, Ortega y García, 2007). La literatura atribuye estas dificultades conceptuales a varios factores. Por un lado, parece ser un contenido que históricamente se ha tratado de una forma teórica y descriptiva (Castelló y Márquez, 2014) en la que el alumno únicamente ha tenido que recordar distintos aspectos que le han sido transmitidos. Por otro lado, nos encontramos con el hecho de que gran parte de los libros de texto hacen alusión casi exclusivamente a un modelo hidro-geológico (ríos, mares y aguas sub-terrráneas) a nivel planetario (Calvo et al., 2007), con el que difícilmente es posible inferir que se trata también de un proceso que se produce a pequeña escala. Además, en el famoso diagrama sobre el ciclo del agua con frecuencia tan sólo representa una parte de los procesos implicados. La condensación, la filtración o la dinámica interna de las nubes suelen ser aspectos confusos e incluso inexistentes. En otras ocasiones el vapor de agua es representado como gotitas (Calvo et al., 2007) o la evaporación es enlazada directamente con la formación de las nubes sin mencionar el proceso de condensación (Márquez y i Plaza, 2007). En este trabajo compartimos la visión del National Research Council (NRC, 2000) cuando señala que la observación y la aplicación en el aula de situaciones cercanas la experiencia facilita a los estudiantes salvar algunos de los obstáculos que surgen para la comprensión científica de determinados contenidos. Entendemos que una posible forma de salvar algunas de las dificultades que este tema plantea es mediante el trabajo de pequeñas actividades basadas en la resolución de problemas (de Miguel et al., 2009; Nebot, 2007) donde el conocimiento es debatido. En este sentido, la literatura aporta una gran variedad de actividades que abordan el ciclo del agua basadas en esta perspectiva. No obstante, los resultados van a depender, en última instancia, de las estrategias y objetivos que el profesorado emplee para guiar los aprendizajes (Crujeiras, 2017).

\section{El conocimiento expresado en el aula}

Desde la didáctica comprender qué es lo que le ocurre al conocimiento nos conduce inevitablemente a investigar la práctica educativa. Aprender forma parte de un proceso de comunicación, principalmente oral, donde se crean significados que son compartidos pudiendo derivar incluso en ideas alternativas (Candela, 1999). El lenguaje es en un vehículo para el desarrollo del pensamiento, de forma que al hablar con otros se produce la construcción de ideas contextuales con múltiples interpretaciones. Especialmente en etapas tempranas como Primaria, el conocimiento que es construido en el aula se origina principalmente de forma oral empleando para ello ciertos términos o palabras. No obstante, los significados otorgados a ciertas palabras no son siempre los mismos para unas personas que para otras, sino que dependen en gran medida del contexto en el que son creados. De esta manera, el conocimiento es un ente que se comparte y sufre modificaciones a través de procesos 
comunicativos (Jorba, Gómez y Prat, 2000). La apropiación y la construcción de significados que se dan dentro de una situación específica social se producen a través de una maraña de observaciones, inferencias y debates. En este sentido, el análisis conversacional del habla espontánea de un aula pone la atención en los detalles, que, de otra manera, por ejemplo, mediante evaluaciones de tipo cuestionario, no reflejaría adecuadamente el trabajo educativo escolar (Candela, 1999).

\section{Objetivo}

El objetivo global de la investigación se enfoca en analizar las ideas expresadas por el alumnado de Primaria durante el desarrollo de dos actividades sobre el ciclo del agua de corte indagador.

\section{Métodos}

\section{Participantes}

El estudio se realiza en una clase de $2^{\circ}$ curso de Primaria de un colegio público con un número de 24 estudiantes.

\section{Instrumento}

Como software de análisis se ha empleado el programa Transana (www.transana.org).

\section{Contexto del estudio}

Las dos actividades se realizan con todo el grupo clase sin que los estudiantes dispongan ningún material de texto para su desarrollo. Como fuente de datos se emplea una videograbación de casi una 1 hora. En la tabla 1 se presenta un resumen detallado de cada actividad y su duración.

Tabla 1. Resumen de las actividades analizadas

\section{Actividad 1 ¿Cómo ha llegado el agua a la planta?}

27:27 La actividad se basa en el uso de un dispositivo que consta de una maceta con tierra seca, un

(min) pequeño tarro de agua depositado en la zona de la tierra (sin contacto con la misma), y un film de plástico transparente que recubre la planta. El montaje realizado queda a la vista de los alumnos y se mantuvo así durante una semana, en un apartado del aula junto a la ventana y sin manipular. Toda la sesión va dirigida al grupo clase.

Actividad 2 Si los ríos van al mar ¿por qué no crece?

30:33 Los alumnos se encuentran sentados en sus correspondientes pupitres mientras que el futuro maestro

(min) expresa a los alumnos una duda que lleva pensando desde hace tiempo. La duda, referida a que si en el mar llueve por lógica debería crecer cada año, es planteada junto a dibujo en la pizarra donde representa el mar, dos montañas y un río entre las dos montañas que desemboca en el mar. Los alumnos aportan explicaciones para dar respuesta. Una vez consensuada la explicación, el futuro maestro en prácticas propone hacer un dibujo entre todos en la pizarra para que posteriormente cada alumno realice uno de forma individual. Toda la sesión va dirigida al grupo clase a excepción de los dos minutos finales en los que los alumnos hacen un dibujo de forma individual.

\section{Procedimiento - Análisis de datos}

Desde la idea de que los estudiantes integran 'pequeños' elementos de conocimiento cuando aprenden, una forma de desvelar estos significados es a través del concepto de faceta. Consideramos que las facetas hacen referencia a producciones verbales que nos permiten la reconstrucción de significados y no del conocimiento (Tiberghien, 2007). De esta forma, nos referimos a una faceta como un pequeño elemento de 
conocimiento o idea que un interlocutor expresa en un enunciado verbal en relación con un concepto científico (Galili y Hazan, 2000). Para la obtención de una faceta el investigador guarda el significado otorgado por el interlocutor, al margen de si éste coincide con el aprobado científicamente. Además, durante el análisis se diferencia si la faceta es construida por el maestro, por el alumno o si es una co-construcción alumno-maestro o alumno-alumno. A continuación, se muestra un ejemplo de creación de facetas:

Alumna 1: Por, porque a la vez suben las gotas esas.

Futuro maestro: $Y$ ¿por qué suben las gotas? Porque, claro...

Alumnos: Porque, porque...

Alumna 1: Cuando hay sol...

Alumnos: Calienta.

Futuro maestro: Muy bien.

Alumna 1: Y se hace gas.

En este diálogo están envueltas tres ideas que se concretan en las siguientes facetas: Las gotas del mar suben, El sol calienta el agua y El agua se hace gas. Una vez analizado todo el discurso a cada faceta se le asigna un grupo temático, así como un código (Tabla 2).
Grupos de facetas
Tabla 2. Ejemplo parcial de la lista facetas de la actividad 1
E: Evaporación
Código Facetas
E1 Las gotas del mar suben
(...) E3 El agua se hace gas
(...) E7 El agua sube en forma de vapor
$\begin{array}{lll}\text { C: Condensación } & \mathrm{C} 1 & \text { Las gotas de vapor de agua condensan y se forman gotitas }(\mathrm{P})\end{array}$
S: Contraste de temperatura S1 El gas se enfría. El vapor de agua sube y se enfría

Elaborada la lista completa de facetas se procede a la codificación de la transcripción. En la medida que se considera que una faceta queda envuelta en una producción oral (maestro o alumno), esta intervención codificada con el correspondiente (Tiberghien, 2007). Durante este proceso de codificación también se ha tenido en cuenta si la faceta es empleada por el alumno o por el maestro o si se trata de una co-construcción del tipo maestro-alumno o alumno-alumno.

\section{Resultados}

\section{Actividad 1: ¿Cómo ha llegado el agua a la planta?}

Al comienzo de actividad la atención gira en torno al montaje de la planta tapada con un plástico. El objetivo que el futuro maestro plantea tiene que ver con la observación del dispositivo, orientando al alumnado para que realicen descripciones sobre el estado físico de la planta, el plástico que la tapa, así como el nivel del agua de la vasija. Es un hecho que han transcurrido unos días durante los cuales la planta ha estado tapada, siendo los datos que el dispositivo ofrece los siguientes:

1. Que la planta parece tener un buen aspecto: color verde y turgente.

2. Que la tierra ahora está mojada.

3. Que el nivel agua del tarro ha descendido que hace unos días, cuando pusieron el montaje.

4. Que han aparecido de gotas en el plástico. 
Las primeras observaciones que insta el futuro maestro a realizar tienen que ver con aspectos físicos de la planta como son el color o el porte. El hecho de que la planta tenga un color verde y esté tiesa se asocia a que ha debido de recibir una buena alimentación, es decir, agua de la tierra y luz (dato 1). Es una alumna la que expone que la planta ha debido de tomar agua por sus pelitos (inferencia 1). Otro dato expresado es que cuando, hace una semana, depositaron el tarro con agua sobre la tierra, y antes de tapar la planta con el film de plástico, ésta estaba seca (dato 2). Sin embargo, después de una semana pueden observar que la tierra está húmeda (dato 3 ) lo que los lleva a considerar la necesidad de un movimiento del agua dentro de la planta (inferencia 2).

Futuro Maestro: Y ahora la tierra está mojada.

Alumna: Porque el vapor como es agua ha bajado y.... al meterse dentro, ha subido un poquito a la superficie.

Futuro Maestro: ¿El agua esta que se ha evaporado y que caído de dónde salía?

Alumno: Del tarro.

Este movimiento es explicado por los alumnos como una consecuencia del calor existente dentro del dispositivo tal y como muestran las siguientes facetas:

- En la planta hace calor.

- El agua se ha ido calentando (inferencia 3).

La primera explicación que justifica el descenso de agua en el tarro es que, al haber hecho calor en la planta, el agua del tarro 'se ha hecho un poco de gas' (inferencia 4) y ese gas ha sido captado por los pelitos de la planta (inferencia 5).

Alumna: Es que el agua en el tarrito suelta un poco de vapor y esa agua y la raíces, esos pelitos lo absorben.

La tierra mojada se interpreta explicando que el agua procedente del tarro ha bajado a la tierra (inferencia 6), aunque el alumnado no diferencia si esa agua se encuentra en estado líquido o vapor ej. El vapor se ha metido dentro de la tierra. Existe una dificultad en diferenciar el paso del estado vapor del agua al estado líquido. La aparición de gotitas en el plástico es explicada por el futuro maestro introduciendo la idea de frío ej. El vapor se ha enfriado.

En primera instancia, los alumnos sólo son capaces de identificar un cambio de estado de líquido a gas (evaporación) utilizando como variable imprescindible el concepto de calor. A diferencia de la evaporación, la condensación es introducida por el futuro maestro, quien indica que el vapor se ha convertido en agua y además introduce el término condensar ej. El agua se ha condensado y baja.

En resumen, podemos concluir que el modelo de cambios de estado del agua basado expresado se basa en las siguientes ideas:

- El agua, según la cantidad de calor, cambia de estado líquido/gas llamado vapor de agua.

- Cuando el agua líquida se calienta se transforma en vapor de agua el cual asciende.

- Cuando el vapor de agua se enfría forma gotas de agua y vuelve a descender. 
La presencia del dispositivo experimental ayuda a los alumnos a comprender que la planta sobrevive gracias a los cambios de estado que experimenta el agua. La planta representa un nivel de conocimiento clave como es relacionar lo que saben sobre las plantas con los cambios fisicoquímicos del agua. De esta forma, la actividad se enfoca hacia la integración de dos modelos teóricos:

a) sobre la necesidad de captar agua para la vida de las plantas y

b) sobre los cambios de estado del agua (que explica que lo anterior sea posible).

A pesar de las posibles deficiencias del modelo de cambios de estado establecido, el diseño experimental sirve a los alumnos para confirmar los datos, jugando un papel fundamental en sus razonamientos. La aportación de datos útiles a través del dispositivo facilita el replanteamiento de las ideas de los alumnos, instándoles a integrar dichos datos en sus explicaciones.

\section{Actividad 2: Si los ríos van a parar al mar y en la playa llueve ¿por qué el mar no crece?}

La segunda actividad se orienta hacia la construcción de un modelo de ciclo del agua a escala geológica. Al inicio de la sesión el futuro maestro representa en la pizarra un río que nace entre dos montañas, desembocando en el mar junto con unas casas a la orilla, mientras que plantea a los alumnos la pregunta ¿Cómo es posible que si los ríos van a parar al mar éste no crezca? Acto seguido borra dicho dibujo mientras los alumnos aportan explicaciones. Posteriormente, el futuro maestro solicita elaborar un dibujo entre todos, el cual realiza él en la pizarra. Este dibujo consta de los siguientes elementos: unas montañas, un río, el mar, unas casas, un sol, las nubes, vapor de agua, rayos del sol y gotitas dentro de la nube. Finalmente, los alumnos deben realizar individualmente un dibujo sobre lo explicado.

Las ideas que dan lugar al conjunto de facetas son elaboradas todas ellas por el alumnado, siendo una gran mayoría relacionadas con la evaporación.

Futuro maestro: $Y$ al calentarlo Andrea ¿qué pasa? ¿Qué le pasa a esa agua?

Alumna: Que se, que se va evaporando.

La evaporación se explica como la transformación de agua líquida mediante la acción del sol. Sin embargo, el paso de gas a líquido se explica a través de la idea de un 'contraste de temperatura', sin emplear para ello el término condensar.

Futuro maestro: ¿Vale? Entonces, cuando sube ¿qué le pasa Andrea?

Alumna: Que, que... se... enfría.

Futuro maestro: Se enfría.

Alumna: Y se hace una nube porque cuando más se caliente más vapor sube, la nube más grande se hace y al final cae nieve o granizo. Pero si sólo sube normal, caerán gotas de agua.

En cuanto al proceso de evaporación, surgen dificultades a la hora de identificar el agua como gotas o como vapor durante su proceso de ascensión.

Futuro maestro: Piensa un poco. Álvaro ayúdale. Cuándo calentamos el agua ¿qué pasa?

Alumno: Sube

Futuro maestro: Sube, pero en forma ¿de qué?

Alumno: De gotas

Futuro maestro: No... 


\author{
Alumno: De vapor \\ Futuro maestro: De vapor...
}

Algo similar ocurre con las nubes cuando los alumnos las definen en unos casos como algo formado por vapor y en otros por agua. Concretamente cuando los estudiantes tratan de explicar cómo se produce el fenómeno de la lluvia hablan de una nube formada por gotas las cuales acaban cayendo cuando existe un elevado peso. Sin embargo, cuando los estudiantes tratan de explicar el proceso de evaporación del agua en el mar hablan de una nube formada exclusivamente por vapor de agua.

Alumna: No lo vemos, no lo vemos no lo vemos porque es gas y el gas no se ve.

Futuro maestro: Ah... Muy bien.

Alumna: Porque está en las nubes.

Futuro maestro: Muy bien. Entonces el sol calienta el agua y el agua se evapora ¿no? Por otro lado, los distintos tipos de precipitación (lluvia o nieve) son explicados en términos de frío, entendiendo que a mayor frío precita en forma de nieve.

Alumno: Que cae

Futuro maestro: Claro, que cae, que precipita ¿vale?

Alumno: Pero cuando tiene mucho más frío caen copos de nieve.

En cuanto a los dibujos elaborados por los alumnos puede verse como en todos ellos realizan se muestra un dibujo en el que aparecen dos nubes a distintas alturas respecto de la montaña. Una nube se sitúa a una altura más baja con gotitas y la otra, situada a una altura más alta con asteriscos. De esta forma una nube que se encuentra a una altura considerable (la de una montaña) precipitará en forma de nieve, mientras que otra que se encuentre a menor altura -menor frío- precipitará en forma líquida (gotas).

Además, se desprende la idea de que la evaporación es un proceso exclusivo al mar mientras que la precipitación lo es de las montañas. De los dibujos elaborados por los estudiantes se aprecia cómo el sol se convierte en un elemento indispensable que actúa como punto de inicio del proceso.

En resumen, podemos concluir que del análisis de las ideas expresadas el modelo hidrogeológico expresado durante la actividad es el siguiente:

- Los ríos bajan por las montañas y vierten agua en el mar.

- El sol calienta el agua y las gotas se transforman en vapor de agua.

- El vapor de agua sube a las nubes, pero no lo vemos porque es gas y el gas no se ve.

- Arriba, en la atmósfera, hace frío, por lo que el vapor de agua se condensa, formando

- gotitas de agua (la nube).

- El viento empuja a las nubes y cambian de tamaño.

- Encima de las montañas, lloverá o nevará o granizará, dependiendo del frío que haga.

- Ambivalencia en la explicación de las precipitaciones. Éstas son explicadas como consecuencia de una nube sometida a frío cuando se trata de diferenciar los distintos tipos de precipitación (nieve, lluvia o granizo) o debido a un elevado peso de las gotitas que la conforman.

- La evaporación como fenómeno que sucede exclusivamente en el mar.

- La precipitación como fenómeno que sucede exclusivamente en las montañas. 


\section{Discusión}

Este tipo de investigación se centra en conocer cómo el saber es aprendido, de manera que los resultados obtenidos permitan acercar al profesorado las dificultades que el alumnado pueda encontrar durante el estudio del ciclo del agua. Desde nuestra perspectiva, consideramos que este tipo de contribuciones pueden ayudar a que los docentes a reconocer situaciones que favorezcan la reflexión sobre los significados en la elaboración de ideas en el aula.

Entre los resultados más reveladores destacamos cómo los estudiantes expresan los conceptos de evaporación y condensación en distintos términos dentro del contexto hidro-geológico (Driver, Guesne y Tiberghien, 1985). El paso de gas a líquido se explica como una consecuencia de un 'contraste de temperatura', mientras que el paso de líquido a gas es asociado a la presencia de un foco caliente (radiador o Sol). Por otro lado, cuando se trata de explicar el cambio de estado de gas a líquido (condensación) dentro del ciclo del agua, éste es explicado a través de una nube que debe chocar con otro elemento (montaña, otra nube...) para que se produzca su transformación en líquido (lluvia). La nube, por tanto, es visualizada como un gas. Así, cuando los alumnos tratan de explicar la condensación en un contexto del ciclo del agua, y no dentro del dispositivo experimental, la idea de 'contraste de temperatura' deja de existir para ser reemplazada por la de 'choque entre dos cuerpos'. En este sentido debemos señalar la necesidad de compresión de un modelo fisicoquímico sólido para la posterior construcción del modelo hidrogeológico del agua.

Para finalizar queremos concluir cómo la reproducción de aspectos parciales del ciclo del agua facilita la reconsideración de sus ideas, así como un debate más profundo en las mentes de los estudiantes sobre los procesos implicados.

\section{Referencias}

Brousseau, G (1988). Le contrat didactique: le milieu. Recherches en Didactique des Mathématiques, 9(3), 309-336.

Bruner, J (1984) Acción, pensamiento y lenguaje. Buenos Aires: Ed. Paidós.

Calvo, M, Reyero, C, Vidal, MP, Ortega, JGM y García, EG (2007). El trabajo con modelos en aguas subterráneas. Enseñanza de las Ciencias de la Tierra, 15(3), 341-347.

Candela, A (1999). Prácticas discursivas en el aula y calidad educativa, Revista Mexicana de Investigación Educativa, 4(8), 273-298.

Cardak, O (2009). Science students' misconceptions of the water cycle according to their drawings. Journal of Applied Sciences, $9(5), 865-873$

Castelló, MRN y Márquez, C (2014). El Ciclo del agua en el laboratorio. Alambique: Didáctica de las ciencias experimentales, $77,17-24$

Crujeiras-Pérez, B (2017). Análisis de las estrategias de apoyo elaboradas por futuros docente de educación secundaria para guiar al alumnado en la indagación. Revista Eureka sobre enseñanza y divulgación de las ciencias, 14(2), 473-486.
De Miguel, A, Lado, JJ, Martínez, V, Leal, M y García, R (2009). El Ciclo hidrológico: experiencias prácticas para su comprensión. Enseñanza de las Ciencias de la Tierra, 17(1), 78-85.

Driver, R, Guesne, E, \& Tiberghien, A (1985). Children's ideas in Science. Glasgow: Milton Keynes, Open University Press.

Galili, I, \& Hazan, A (2000). Learners' knowledge in optics: interpretation, structure and analysis. International Journal of Science Education, 22(1), 57-88.

Jorba, J., Gómez, I. \& Prat, À. (Eds.). (2000). Hablar y escribir para aprender: uso de la lengua en situación de enseñanzaaprendizaje desde las áreas curriculares. Editorial Síntesis.

Lemke, J. (1997). Aprender a hablar ciencia. Lenguaje, aprendizaje y valores. Barcelona: Paidós.

Márquez, C, Izquierdo, M y Espinet, M (2003). Comunicación multimodal en la clase de ciencias: el Ciclo del agua. Enseñanza de las ciencias: revista de investigación y experiencias didácticas, 21(3), 371.

Márquez, C y Plaza, JB (2007). Una propuesta de análisis de las representaciones de los alumnos sobre el ciclo del agua. Enseñanza de las Ciencias de la Tierra, 15(3), 280-286. 
Márquez, C, Izquierdo, M y Espinet, M (2006). Multimodal science teachers' discourse in modeling the water cycle. Science Education, 90(2), 202-226.

National Research Council. (2000). Inquiry and the national science education standards: A guide for teaching and learning. National Academies Press.

Nebot, MR y Márquez, C (2014). El Ciclo del agua en el laboratorio. Una propuesta de modelización. Alambique Didáctica de las Ciencias Experimentales, 77,17-24.

Nebot, R (2007). El ciclo del agua en una garrafa. Enseñanza de las Ciencias de la Tierra, 15(3), 333-340.

Rivero García, A, Solís Ramírez, E, Porlán Ariza, R, Azcárate Goded, MDP y Martín del Pozo, R (2017). Cambio del conocimiento sobre la enseñanza de las ciencias de futuros maestros. Enseñanza de las Ciencias, 35(1), 29-52.
Sensevy, G (2011). Le sens du savoir. Éléments pour une théorie de l'action conjointe en didactique. Bruxelles: de Boeck.

Smith, C, Wiser, M, Anderson, CW, \& Krajcik JS (2006) Implications of Research on Children's Learning for Standards and Assessment: A Proposed Learning Progression for Matter and the Atomic Molecular Theory Measurement: Interdisciplinary Research and Perspective, 14(1\&2), 1-98.

Tiberghien, A (2007). Construction of students' knowledge in relation to a teaching sequence: hypotheses on learning and knowledge, (pp.1-16). Jerusalem: ESERA 2009 Conference.

Vílchez González, JM y Bravo Torija, B (2015). Percepción del profesorado de ciencias de educación primaria en formación acerca de las etapas y acciones necesarias para realizar una indagación escolar. Enseñanza de las Ciencias, 33(1), 185-202. 